PATIENT SAFETY

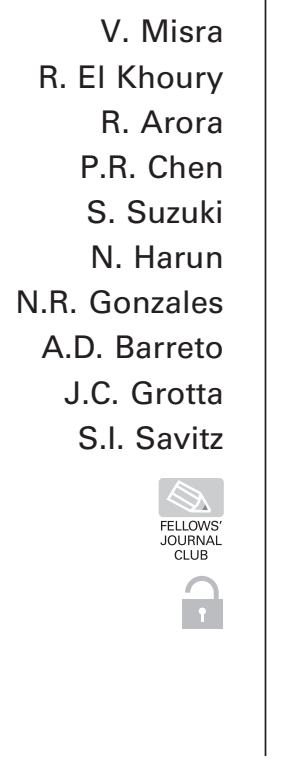

\title{
Safety of High Doses of Urokinase and Reteplase for Acute Ischemic Stroke
}

BACKGROUND AND PURPOSE: ET is considered in selected patients with AIS with persistent arterial occlusion after receiving IVT. Limited data exist on the safety of IA high doses of UK and RT for ET. We investigated any correlation between IA doses of UK or RT and safety outcomes in patients who underwent ET.

MATERIALS AND METHODS: We identified all patients from our stroke registry who received UK or RT for ET from 1998 to 2008. Demographics, baseline National Institutes of Health Stroke Scale scores, recanalization rates, rates of attempted $\mathrm{MT}$, mortality, $\mathrm{SICH}$, and discharge modified Rankin Scale scores were collected.

RESULTS: Of 197 patients; 72 received UK and 125 received RT. More than $90 \%$ of patients in both groups had received prior IVT. The median IA dose of UK was 200,000 U (range, 25,000-1,500,000 U) and of RT was $2 \mathrm{mg}$ (range, 1-8 mg). Concurrent MT was attempted in $59.7 \%$ of UK-treated patients and $72.0 \%$ of RT-treated patients, with $\mathrm{SICH}$ rates of $4.2 \%$ and $8.0 \%$, respectively. Logistic regression adjusting for prior IVT and MT revealed no correlation between $\mathrm{SICH}$ and doses of UK (OR, 1.00; 95\% $\mathrm{Cl}, 0.99-1.00 ; P=.94)$ or RT (OR, $0.803 ; 95 \% \mathrm{Cl}, 0.48-1.33 ; P=.39)$. There was no correlation between mortality and doses of UK (OR, 1.00; 95\% Cl, 0.99-1.00; $P=.51)$ or RT (OR, 1.048; $95 \% \mathrm{Cl}$, $0.77-1.42 ; P=.75)$.

CONCLUSIONS: High IA doses of UK and RT may be safe when given with or without MT in patients with AIS despite receiving a full dose of intravenous recombinant tissue plasminogen activator. These results need prospective validation.

$\mathbf{E}$ $\mathrm{T}$ is considered in selected patients with AIS who have persistent arterial occlusion after receiving IVT. The ability to achieve successful recanalization with reperfusion therapies is the best predictor of good outcome in patients with large artery occlusion. ${ }^{1}$ Given the low recanalization rates associated with IVT, ${ }^{2}$ a substantial number of patients with large artery occlusions receive ET. After the PROACT II study, the AHA/ASA guidelines concluded that IA prourokinase is an option for the treatment of select patients with MCA occlusion within 6 hours of onset, after treatment with the standard of care IVT. ${ }^{3}$ The FDA did not approve prourokinase, and it is not clinically available for use as an IA thrombolytic agent. ${ }^{3}$ Other agents that have been used in IA therapy include UK,

Received August 1, 2010; accepted after revision October 15.

From the Departments of Neurology (V.M., R.E.K., N.H., N.R.G., A.D.B., J.C.G., S.I.S.), Neurosurgery (P.R.C.), and Radiology (S.S.), The University of Texas Medical School at Houston, Houston, Texas; and Department of Neurology (R.A.), Drexel University College of Medicine, Philadelphia, Pennsylvania.

This study was supported by the National Institutes of Health grants T32NS04712 and P50 NS044227.

Please address correspondence to Sean I. Savitz, MD, Department of Neurology, The University of Texas Medical School at Houston, 6431 Fannin St, MSB 7.044, Houston, TX 77030; e-mail: sean.i.savitz@uth.tmc.edu

Indicates open access to non-subscribers at www.ajnr.org

DOI 10.3174/ajnr.A2427
RT, rtPA, and tenecteplase. Prior small case series have demonstrated that IA therapy with thrombolytic agents, ${ }^{4,5} \mathrm{MCD}^{6}$ and a combination of IA thrombolytic agents with $\mathrm{MCD}^{7,8}$ are safe and effective with and without prior full-dose IVT in restoring flow in acute large artery occlusions. A recent metaanalysis concluded that prior full-dose IVT is superior to lowdose IVT in achieving higher recanalization rates and better outcomes when combined with ET. ${ }^{9}$ In a small case series, IA thrombolysis with RT demonstrated a trend toward higher rates of recanalization and SICH without prior IVT compared with IA UK. ${ }^{10}$ The safety, however, of using high IA doses of thrombolytic agents when used with multimodal ET is unclear. We performed a retrospective analysis investigating the relationship between IA dosage of 2 commonly used thrombolytic agents (UK and RT) with SICH and mortality in patients with AIS who underwent ET for symptomatic arterial occlusion.

\section{Materials and Methods}

\section{Patients and Techniques}

We identified 251 patients from our prospective stroke registry who underwent ET for acute anterior circulation ischemic stroke between 1998 and 2008. The study protocol was approved by the institutional review board before initiation. All patients were evaluated by an experienced stroke team made up of vascular neurologists and fellows at 
a Joint Commission on Accreditation of Healthcare Organizationscertified comprehensive stroke center. A baseline CT head and vascular imaging (CT angiogram or transcranial Doppler) was performed on all patients before undergoing ET. All patients received treatment with full-dose IVT, if eligible as per AHA/ASA guidelines. The decision to proceed with IA therapy was jointly made by the stroke team in consultation with an experienced neuroendovascular service comprising an interventional neurologist, an endovascular neurosurgeon, or interventional neuroradiologists. The choice of the IA thrombolytic agent was based on availability, and the dose used was at the discretion of the interventionalist. UK was available from 1998 to 2000 and RT from 2001 to 2003 . Then, both agents were available from 2003 to 2006. RT was subsequently used after 2006 until 2008. The agents were never used together or in combination with another thrombolytic agent. No patient received IA UK or RT beyond 6 hours from symptom onset or any glycoprotein IIb/IIIa inhibitors during the procedure. All patients also received intravenous heparin infusion to maintain activated clotting time twice the baseline value. MT including MCD with microwire, snare, balloon angioplasty, and stent placement were performed as deemed appropriate by the interventionalist. Mechanical embolus retrieval was performed by using Merci retriever (Concentric Medical, Mountain View, California) and Penumbra System (Penumbra, Alameda, California) following FDA approval. Patients who underwent stent placement did receive antiplatelet loading through a nasogastric tube during the procedure. The choice of the antiplatelet agent was jointly made by the neurointerventionalist and the vascular neurologist considering the individual patient's prior antiplatelet medication use. All patients were subsequently admitted to the neuroscience-intensive care unit or stroke unit and were managed by the stroke team as per standard guidelines. ${ }^{3}$ The interventional neuroradiologist analyzed all the angiograms by using TICI scale as described previously. ${ }^{6}$ Good recanalization was defined as the final TICI score $\geq 2 \mathrm{~b}$. CT or MR imaging brain scans were performed 24 hours after treatment or in case of any neurologic deterioration (defined as $\geq 2$-point increase in the NIHSS) and were evaluated by the neuroradiologist or the vascular neurologist. The NIHSS score was again determined at 24 hours. SICH was defined as a $\geq 2$-point increase in the NIHSS that could be attributed to the intracranial hemorrhage. The baseline patient demographics, stroke risk factors, presentation, 24-hour NIHSS scores, mortality, and discharge mRS scores were extracted from the stroke registry. We excluded patients who were enrolled in clinical trials from this study.

\section{Statistical Analysis}

Data are presented as median (range) and mean with SD where appropriate. Multivariate analysis with logistic regression was performed controlling for confounding factors to investigate association and obtain ORs with $95 \%$ CIs. A $P$ value of $\leq .05$ was used to determine statistical significance. The data were analyzed by using SAS, version 9.2 (SAS Institute, Cary, North Carolina).

\section{Results}

\section{Patient Demographics and Clinical Characteristics}

We identified 197 patients who received IA reperfusion therapy with pharmacologic agents; 72 received UK and 125 received RT within 6 hours of the onset of symptoms. Both groups had similar age, sex distribution, and stroke risk factors (Table 1). The median NIHSS values at presentation were 16 (range, 3-27) in the UK group and 18 (range, 2-39) in the RT

\begin{tabular}{lcc}
\hline \multicolumn{3}{l}{ Table 1: Patient demographics and clinical characteristics } \\
\hline & UK $(n=72)$ & $\mathrm{RT}(n=125)$ \\
\hline Age (mean \pm SD), yr & $58 \pm 15$ & $61 \pm 13$ \\
Male & $36(50.0)$ & $67(53.6)$ \\
Female & $36(50.0)$ & $58(46.4)$ \\
Hypertension & $43(59.7)$ & $78(62.4)$ \\
Diabetes mellitus & $14(19.4)$ & $20(16.0)$ \\
Coronary artery disease & $13(18.0)$ & $33(26.4)$ \\
Atrial fibrillation & $14(19.4)$ & $29(23.2)$ \\
Tobacco use & $11(15.3)$ & $26(20.8)$ \\
Blood glucose (mean \pm SD), mg/dl & $149.4 \pm 55$ & $134.0 \pm 51$ \\
Presentation NIHSS median (range) & $16(3-27)$ & $18(2-39)$ \\
Prior IV rtPA treatment $(n=182)$ & $65(90.3)$ & $117(93.6)$ \\
\hline
\end{tabular}

Note:-Values in parentheses are percentages, except for Presentation NIHSS median.

\begin{tabular}{|c|c|c|}
\hline & UK $(n=72)$ & $\mathrm{RT}(n=125)$ \\
\hline ICA occlusion & $22(30.5)$ & $50(40.0)$ \\
\hline $\begin{array}{l}\text { Carotid-t } \\
\text { occlusion }\end{array}$ & $8(11.0)$ & $23(18.4)$ \\
\hline MCA occlusion & $50(69.5)$ & $75(60.0)$ \\
\hline M1 occlusion & $32(44.5)$ & $45(36.0)$ \\
\hline M2 occlusion & $18(25.0)$ & $30(24.0)$ \\
\hline $\begin{array}{l}\text { IA thrombolytic dose } \\
\text { (median, range) }\end{array}$ & $200,000 \cup(25,000-1,500,000 \mathrm{U})$ & $2 \mathrm{mg}(1-8 \mathrm{mg})$ \\
\hline $\begin{array}{l}\text { Mechanical } \\
\text { thrombolysis }\end{array}$ & $43(59.7)$ & $90(72.0)$ \\
\hline $\begin{array}{l}\text { Clot disruption } \\
\text { with microwire }\end{array}$ & 18 & 37 \\
\hline Snare & 2 & 3 \\
\hline Balloon & 3 & 7 \\
\hline Balloon + stent & 5 & 6 \\
\hline Merci & 13 & 30 \\
\hline Penumbra & 0 & 3 \\
\hline $\begin{array}{l}\text { Merci }+ \\
\quad \text { Penumbra }\end{array}$ & 0 & 3 \\
\hline $\begin{array}{l}\text { Merci + balloon } \\
\quad+\text { stent }\end{array}$ & 2 & 1 \\
\hline
\end{tabular}

Note:-Values in parentheses are percentages, except for IA thrombolytic dose.

group. One patient in each group presented with minor symptoms (NIHSS, 3 and 2 in the UK and RT group, respectively), with subsequent neurologic worsening, and they were then treated with IVT and ET. More than $90 \%$ of the patients in both groups received prior IVT $(90.3 \%$ in UK group and $93.6 \%$ in RT group).

\section{Angiographic and Procedural Characteristics}

The angiographic findings and procedural characteristics are shown in Table 2. Emergent diagnostic angiography revealed internal carotid artery occlusion in $22(30.5 \%)$ patients in the UK group and in $50(40 \%)$ patients in the RT group. MCA occlusion was seen in $50(69.5 \%)$ and $75(60 \%)$ patients in the UK and RT group, respectively. The median IA dose of UK was 200,000 U (range, 25,000-1,500,000 U) and of RT was $2 \mathrm{mg}$ (range, 1-8 mg), respectively. MT was attempted in $59.7 \%$ and $72.0 \%$ of the UK and RT group, respectively (Table 2).

\section{Angiographic and Clinical Outcomes}

Good recanalization ( $\mathrm{TICI} \geq 2 \mathrm{~b}$ ) was seen in 40 (55.5\%) patients in the UK group and $77(61.6 \%)$ patients in the RT group. The median NIHSS values at 24 hours were 12 (range, $0-34$ ) in the UK group and 15 (range, $0-39$ ) in the RT group. 


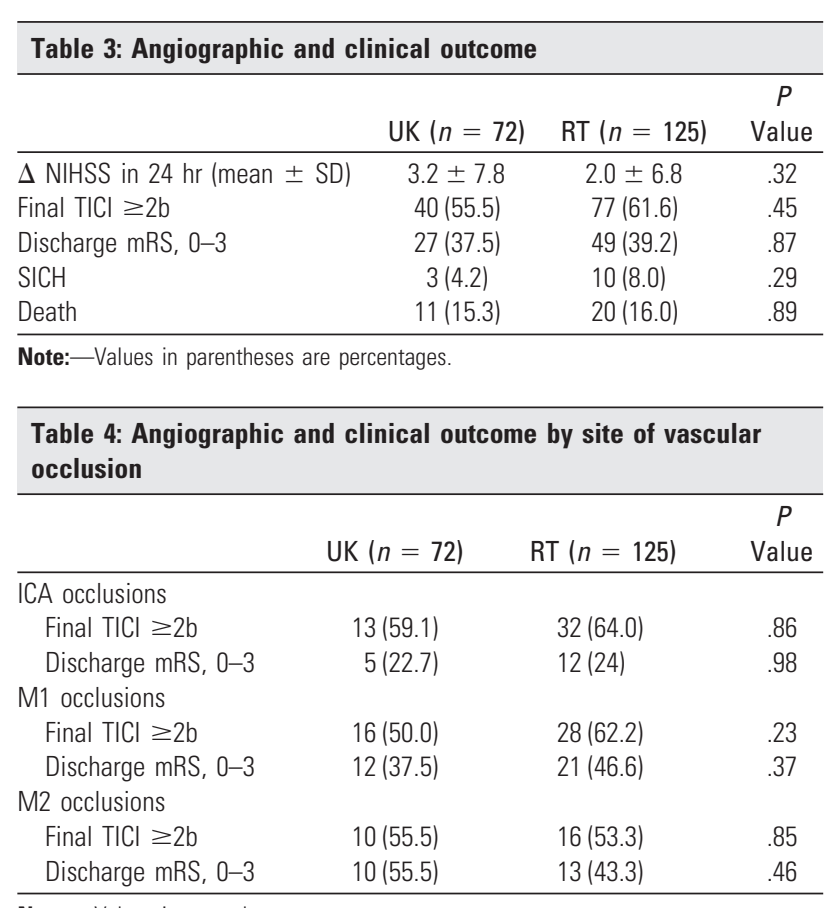

Note:-Values in parentheses are percentages.

There was no difference in the 24-hour outcomes (change in NIHSS score between preprocedure and at 24 hours) and discharge functional outcomes (discharge mRS, $0-3$ ) or in mortality in the 2 groups (Table 3 ). There was also no difference in the recanalization rates and discharge outcomes by sites of arterial occlusion in the 2 groups (Table 4 ).

$\mathrm{SICH}$ was seen in $3(4.2 \%)$ and $10(8.0 \%)$ patients in the UK and RT group, respectively, but SICH was not statistically significant. Logistic regression analysis adjusting for prior IVT and MT revealed no correlation between SICH and dose of UK (OR, 1.00; 95\% CI, 0.99-1.00; $P=.94$ ). There was also no correlation between SICH and dose of RT (OR, 0.803; 95\% CI, $0.48-1.33 ; P=.39$ ). The average dose of UK in patients who developed SICH $(295,000 \pm 212,896 \mathrm{U}$ [range, 75,000$500,000 \mathrm{U}])$ was similar to those without SICH $(286,533 \pm$ $312,256 \mathrm{U}$ [range, $25,000-1,500,000 \mathrm{U}$ ], $P=.96$ ). Patients who developed SICH after IA therapy with RT also received similar doses (2.4 $\pm 1.71 \mathrm{mg}$ [range, $1-7 \mathrm{mg}])$ compared with those who did not develop SICH $(2.81 \pm 1.53 \mathrm{mg}$ [range, $1-8$ $\mathrm{mg}], P=.42)$. There was also no correlation between mortality and doses of UK (OR, 1.00; 95\% CI, $0.99-1.00 ; P=.51)$ or RT (OR, $1.048 ; 95 \%$ CI, $0.77-1.42 ; P=.75)$. We also found no correlation between discharge mRS of $0-3$ and doses of UK (OR, 1.00; 95\% CI, $0.99-1.00 ; P=.15$ ) or RT (OR, 1.12; 95\% CI, $0.88-1.43 ; P=.36)$.

\section{Discussion}

MT in combination with IA pharmacologic thrombolysis has been associated with higher rates of recanalization. ${ }^{11}$ Multimodality ET using evolving techniques including mechanical embolus retrieval with newer FDA-approved devices such as Merci and the Penumbra System is being increasingly used in achieving recanalization in patients with persistent large artery occlusion after standard IVT. Before FDA approval of these devices, IA pharmacologic fibrinolysis was the mainstay of ET in combination with MCD by using microwire, snare, or with balloon angioplasty in very few patients. Full-dose IVT is more efficacious than low-dose IVT when combined with ET in achieving better recanalization rates and clinical outcomes. Nine IA thrombolytic agents, when used in low doses, have been found to be safe in conjunction with $\mathrm{MCD} .^{6-8}$ However, in a series of 43 patients, an IA UK dose $>1,500,000 \mathrm{U}$ was implicated as a predictor of serious complications. ${ }^{12} \mathrm{~A}$ series of 8 patients suggested that an IA rtPA dose up to $40 \mathrm{mg}$ is safe, ${ }^{13}$ but these patients did not receive prior IVT. In a retrospective study of 185 patients, combination of IVT and IA UK was found to be associated with the development of parenchymal hematomas after multimodal ET. ${ }^{14}$ However, this series had 4 patients receiving both IVT and IA UK and 10 patients receiving both IVT and any IA thrombolytic agent. We believe there is a tendency to minimize the use of IA fibrinolytic agents after systemic thrombolysis, more so if MT is being performed to achieve recanalization, due to perceived risks of SICH.

Achieving complete recanalization being the eventual goal of ET, MT by using FDA-approved devices will be increasingly used to treat patients with large artery occlusions, given the higher recanalization rates associated with this technique. ${ }^{1,11}$ However, the presence of residual clot in the distal smaller vessels or dislodgement of partially fragmented clot after embolus retrieval presents a challenge during the interventional procedure. Mechanical embolus retrieval does recanalize the occluded larger arteries without considering the status of the distal smaller arteries. It also has been suggested that recanalization of the primary arterial occlusive lesion does not necessarily translate into reperfusion of ischemic tissue through the distal capillaries. ${ }^{15}$ Other mechanical techniques, including MCD by using microwire, could be unsafe when performed in small distal arteries and are unlikely to be of any additional benefit in restoring flow to the ischemic tissue. IA pharmacologic fibrinolysis remains the only possible alternative in such situations to ensure complete angiographic reperfusion to the ischemic tissue. In our study, we found no correlation between the dose of IA UK or RT and subsequent development of SICH and mortality. The doses of these agents were also similar in patients with or without SICH. Patients in our study who received RT were also more likely to be treated with mechanical embolus retrieval by using Merci and the Penumbra System. This could be explained by the unavailability of UK after 2006 and the FDA-approved Merci in 2004 and Penumbra System more recently in 2008. Given that systemic rtPA at a dose of 0.9 $\mathrm{mg} / \mathrm{kg}$ remains the only proved therapy for treatment of acute stroke, ${ }^{3}$ it is important to treat all eligible patients with IVT before initiating ET in selected patients. In our study, $>90 \%$ patients receiving either UK or RT also received IVT, and prior IVT was not associated with any increase in SICH or mortality.

Fibrinolytic agents have been used in thrombo-embolic disorders other than ischemic stroke with variable efficacy. UK, RT, and rtPA have demonstrated equivalent efficacy and safety in catheter-directed thrombolysis for deep vein thrombosis, with UK being more expensive than RT and rtPA. ${ }^{16}$ There is also significant variability among various thrombolytic agents in terms of safety and efficacy, depending on dosing regimens when used in endovascular treatment of peripheral artery occlusions. ${ }^{17}$ In AIS studies, the Middle Cerebral Artery Embolism Local Fibrinolytic Intervention Trial used IA UK doses $<1,000,000 \mathrm{U}^{18}$ on the basis of a small case series of 
patients with acute MCA occlusions treated long before the availability of IVT. ${ }^{19} \mathrm{UK}$ is no longer available for use in the United States, and current ongoing clinical trials are using rtPA. The ongoing Interventional Management of Stroke (IMS) III trial limits the IA dose of rtPA to $22 \mathrm{mg} .{ }^{20}$ Furthermore, patients randomized to receive ET in the IMS III trial receive two-thirds the standard FDA-approved dose of IVT. The Mechanical Retrieval and Recanalization of Stroke Clots Using Embolectomy trial also limits the IA dose of rtPA to a maximum of $14 \mathrm{mg}$ regardless of prior full-dose IVT. ${ }^{21}$ Our study demonstrates that high IA doses of thrombolytic agents are safe when used even in conjunction with prior full-dose IVT. Investigators may want to take into consideration that limiting the dose of IV rtPA before ET may not be necessary in future clinical trials. Our data also support investigating the efficacy of IA dose escalation regimens in conjunction with MT in patients with symptomatic intracranial large artery occlusions. Because there is no dose equivalence between different thrombolytic agents, it is difficult to compare UK and RT. However, in our study, we found no differences in angiographic and clinical outcomes between UK and RT over a wide dose range, regardless of the site of intracranial arterial occlusion.

This study is limited by its retrospective design and the results need prospective validation. Unlike a randomized clinical trial, there is variability in the endovascular procedural steps because the dose of IA thrombolytic agent and the selection of MT technique as well as choice of the embolectomy device were at the discretion of the neurointerventionalist. The study population includes patients treated from 1998 to 2008 and the angiograms were analyzed by the interventional neuroradiologists through subsequent years, and this may have introduced a bias. Furthermore, because $>90 \%$ of the patients in this study received prior IVT, only a few patients received IA fibrinolysis alone, which limits drawing any clear conclusions between UK and RT groups. Finally, there have been significant advancements in endovascular technology since the initial use of fibrinolytic agents; however, IA pharmacologic thrombolysis remains an important modality for achieving reperfusion in the distal vasculature inaccessible to mechanical devices.

\section{Conclusions}

Although UK is no longer available, our study supports the safety of high IA doses of 2 pharmacologic agents, UK and RT, in conjunction with MT and prior full-dose IVT. Ongoing prospective clinical trials should explore the role of high IA doses of thrombolytic agents as a means of achieving adequate reperfusion in conjunction with MT in patients with symptomatic intracranial large artery occlusions after full-dose IVT.

\section{References}

1. Nogueira RG, Liebeskind DS, Sung G, et al. Predictors of good clinical outcomes, mortality, and successful revascularization in patients with acute ischemic stroke undergoing thrombectomy: pooled analysis of the Mechanical Embolus Removal in Cerebral Ischemia (MERCI) and Multi MERCI trials. Stroke 2009;40:3777-83

2. Saqqur M, Molina CA, Salam A, et al. Clinical deterioration after intravenous tissue plasminogen activator treatment: a multicenter transcranial Doppler study. Stroke 2007;38:69-74

3. Adams HP, del Zoppo G, Alberts MJ, et al. Guidelines for the early management of adults with ischemic stroke: a guideline from the American Heart Association/American Stroke Association Stroke Council, Clinical Cardiology Council, Cardiovascular Radiology and Intervention Council, and the Atherosclerotic Peripheral Vascular Disease and Quality of Care Outcomes in Research Interdisciplinary Working Groups: the American Academy of Neurology affirms the value of this guideline as an educational tool for neurologists. Stroke 2007;38:1655-711

4. Shaltoni HM, Albright KC, Gonzales NR, et al. Is intra-arterial thrombolysis safe after full-dose intravenous recombinant tissue plasminogen activator for acute ischemic stroke? Stroke 2007;38:80-84

5. Lee KY, Kim DI, Kim SH, et al. Sequential combination of intravenous recombinant tissue plasminogen activator and intra-arterial urokinase in acute ischemic stroke. AJNR Am J Neuroradiol 2004;25:1470-75

6. Noser EA, Shaltoni HM, Hall CA, et al. Aggressive mechanical clot disruption: a safe adjunct to thrombolytic therapy in acute stroke? Stroke 2005;36:292-96

7. Yoon W, Park MS, Cho KH. Low-dose intra-arterial urokinase and aggressive mechanical clot disruption for acute ischemic stroke after failure of intravenous thrombolysis. AJNR Am J Neuroradiol 2010;31:161-64

8. Qureshi AI, Siddiqui AM, Suri MF, et al. Aggressive mechanical clot disruption and low dose intra-arterial third-generation thrombolytic agent for ischemic stroke: a prospective study. Neurosurgery 2002;51:1319-27

9. Georgiadis AL, Memon MZ, Shah QA, et al. Comparison of partial $(.6 \mathrm{mg} / \mathrm{kg})$ versus full-dose $(.9 \mathrm{mg} / \mathrm{kg})$ intravenous recombinant tissue plasminogen activator followed by endovascular treatment for acute ischemic stroke: a metaanalysis. J Neuroimaging 2009; Epub ahead of print

10. Sugg RM, Noser EA, Shaltoni HM, et al. Intra-arterial reteplase compared to urokinase for thrombolytic recanalization in acute ischemic stroke. AJNR Am J Neuroradiol 2006;27:769-73

11. Lin R, Vora N, Zaidi S, et al. Mechanical approaches combined with intraarterial pharmacologic therapy are associated with higher recanalization rates than either intervention alone in revascularization of acute carotid terminus occlusion. Stroke 2009;40:2092-97

12. Tirschwell DL, Coplin WM, Becker KJ, et al. Intra-arterial urokinase for acute ischemic stroke: factors associated with complications. Neurology 2001;57: $1100-03$

13. Qureshi AI, Suri MF, Shatla AA, et al. Intraarterial recombinant tissue plasminogen activator for acute ischemic stroke: an accelerating dose regimen. Neurosurgery 2000;47:473-76

14. Vora NA, Gupta R, Thomas AJ, et al. Factors predicting hemorrhagic complications after multimodal reperfusion therapy for acute ischemic stroke. AJNR Am J Neuroradiol 2007;28:1391-94

15. Soares BP, Chien JD, Wintermark M. MR and CT monitoring of recanalization, reperfusion, and penumbra salvage: everything that recanalizes does not necessarily reperfuse! Stroke 2009;40:S24-27

16. Grunwald MR, Hofmann LV. Comparison of urokinase, alteplase, and reteplase for catheter-directed thrombolysis of deep venous thrombosis. J Vasc Interv Radiol 2004;15:347-52

17. Robertson I, Kessel DO, Berridge DC. Fibrinolytic agents for peripheral artery occlusion. Cochrane Database Syst Rev 2010;3:CD001099

18. Ogawa A, Mori E, Minematsu K, et al. Randomized trial of intraarterial infusion of urokinase within $\mathbf{6}$ hours of middle cerebral artery stroke: the middle cerebral artery embolism local fibrinolytic intervention trial (MELT) Japan. Stroke 2007;38:2633-39

19. Mori E, Tabuchi M, Yoshida T, et al. Intracarotid urokinase with thromboembolic occlusion of the middle cerebral artery. Stroke 1988;19:802-12

20. Khatri P, Hill MD, Palesch YY, et al. Methodology of the Interventional Management of Stroke III trial. Int J Stroke 2008;3:130-37

21. MR and recanalization of stroke clots using embolectomy (MR RESCUE). http://www.clinicaltrials.gov/ct2/show/NCT00389467?term=MR+RESCUE + TRIAL\&rank=1. Accessed July 1, 2009 